\title{
The length dependence of the series elasticity of pig bladder smooth muscle
}

\author{
R. VAN MASTRIGT \\ Department of Urology, Erasmus University, Rotterdam, PO Box 1738, 3000 DR Rotterdam, The Netherlands
}

Received 2nd June 1988

\begin{abstract}
Summary
Strips of urinary bladder smooth muscle were subjected to a series of quick release measurements. Each measurement consisted of several releases and resets to the original length, made during one contraction. The complete length-force characteristic of series elasticity was quantified by estimating $H$, the amplitude of quick release necessary to reduce the active force to exactly zero, and $D_{b}$, a measure for the deviation of the characteristic from a straight line. By measuring a series of contractions at increasing stretched strip lengths, the length dependence of these parameters was studied. It was found that $H$ depends linearly on stretched strip length. On average $H /$ length amounted to 0.04 . $D_{\mathrm{b}}$ decreased when strips were stretched, i.e. a straight line was more closely approximated. Both parameter dependencies support the concept of two separate elastic mechanisms, a linear true passive elasticity in series with a non-linear elasticity in the cross-bridges. For the latter, $H$ amounts to $3.8 \%$ of the initial strip length.
\end{abstract}

\section{Introduction}

Quick release is one of the traditional techniques of investigating the contractile properties of muscle. Even if equipment with an electronically controlled length changer is used, very often the traditional measurement scheme is followed, in which one release is applied at one preset load level, yielding one data point for each contraction. In smooth muscle, especially that of the urinary bladder, the number of contractions which can be measured on one preparation is limited (van Mastrigt \& Glerum, 1985; van Mastrigt et al., 1986). During a contraction the active state changes relatively slowly and quick releases measured during various phases of a contraction can be normalized simply (van Mastrigt \& Tauecchio, 1982). It is, therefore, possible to measure several quick releases during one contraction, and hence to estimate parameters describing the crossbridge interaction in the smooth muscle cell from a single contraction, and to study the variations in the parameters in different circumstances. The present study describes a first analysis of a set of measurements on strips of smooth muscle from the pig bladder, taken at increasing stretched lengths.

\section{Materials and methods}

Fresh pig bladders from the local slaughterhouse were transported to the laboratory in cold Krebs (1932) solution. A strip of approximately $10 \times 23 \mathrm{~mm}$ was cut from the posterior wall of the bladder and mounted in a container containing Krebs solution equilibrated with $95 \% \mathrm{O}_{2}, 5 \%$ $\mathrm{CO}_{2}$ at $37^{\circ} \mathrm{C}$. The solution had the following composition in mmol 1-1: $\mathrm{NaCl} 118 ; \mathrm{KCl} 4.7 ; \mathrm{NaHCO}_{3} 25 ; \mathrm{KH}_{2} \mathrm{PO}_{4}$ 1.2; $\mathrm{CaCl}_{2} 1.8 ; \mathrm{MgSO}_{4} 1.18$ and glucose 11 . One end of the strip was fixed to a Sensotec load cell with a silk thread. This transducer had a resonance frequency of $4.7 \mathrm{kHz}$, a range of $10 \mathrm{~N}$, and a compliance of $9 \mu \mathrm{m} \mathrm{N}^{-1}$. The other end of the strip was mounted in a clamp with penetrating pins which could be moved with a pneumatic cylinder. A 2-mm movement of the clamp could be effected in $10 \mathrm{~ms}$. Bearing in mind that there is a difference in the $V_{\max }$ of striated and smooth muscle in the order of a factor of 30 (Murphy, 1976) this compares to the time resolution common in similar work on striated muscle. Replacing the muscle strip by a silk thread identical to the one used for connecting it yielded an overall compliance of $156 \mu \mathrm{m} \mathrm{N}^{-1}$. From this value the compliance of the set-up plus that of the length of thread tied to the muscle was estimated as less than $60 \mu \mathrm{m}$ $\mathbf{N}^{-1}$. The excursion of the penumatic cylinder was limited by a large plastic disc with a thickness varying in steps of $0.1 \mathrm{~mm}$. The disc could be rotated by a stepper motor. Its position and thus the amplitude of the movement forced by the pneumatic cylinder was monitored by an absolute rotation transducer. Electrical stimulation was applied using two stainless steel electrodes of $20 \times 70 \mathrm{~mm}$, set $25 \mathrm{~mm}$ apart, and running parallel to the strip on both sides. Alternating pulses of $20 \mathrm{~V}, 10 \mathrm{~ms}$ at a repetition frequency of $100 \mathrm{~Hz}$ were obtained from a pulse generator, a special pulse converter and an operational power amplifier. The signal from the load cell was amplified by a strain gauge amplifier with cut-off frequency of $1.4 \mathrm{kHz}$ and fed into a Digital Equipment Professional 350 computer 
using and A/D converter. The signal was sampled at $10 \mathrm{~Hz}$, except during a quick release, when the sampling rate was switched to $1 \mathrm{kHz}$ for $1 \mathrm{~s}$. The computer also controlled the mechanical set-up by rotating and checking the position of the stepper motor, triggering the movement of the pneumatic cylinder and switching electrical stimulation on and off. Following equilibration for $15 \mathrm{~min}$, strips were slowly stretched until a tension of $0.05 \mathrm{~N}$ was attained, and again left to equilibrate for $15 \mathrm{~min}$. Measurements were then made at intervals of $15 \mathrm{~min}$. Each measurement consisted of a maximum of seven quick releases during a period of electrical stimulation. Each release was followed by a reset to the original length after $0.7 \mathrm{~s}$. Releases were applied as soon as the (isometric) force produced by electrical stimulation ceased to rise further. There was a waiting interval of $1 \mathrm{~s}$ between successive releases and electrical stimulation was generally continued until a previously specified number of releases (less than 8 ) had been completed, but a maximum stimulation duration was also specified to prevent fatigue of the preparation in case of errors. Figure 1 shows an example of a contraction with seven releases. A program of amplitudes for the releases was specified before each measurement. The program was adapted in such a way that, according to the results of previous measurements, the largest release was expected to reduce the active force to approximately zero. This program was executed twice, once with the amplitudes in ascending order, and once (during a second contraction, 15 min later) in descending order. Before making measurements during a contraction, the same program of length changes was applied with the same time schedule to the unstimulated muscle, in order to be able to compensate for changes in passive force. Following the measurement of two contractions and associated passive force changes in this way, the length of the strip was manually increased.

The minimum force attained during the release was normalized and compensated for passive force changes by calculating:

$$
F_{\text {rel }}=\frac{F_{\mathrm{dl}}-F_{\text {pasdl }}}{F_{\text {trig }}-F_{\text {pas }}}
$$

where $F_{\mathrm{dl}}$ is the minimum force in quick release during electrical stimulation; $F_{\text {pasdl, }}$ the minimum force in quick release without electrical stimulation; $F_{\text {trig, the average }}$ force level $50 \mathrm{~ms}$ before quick release during electrical stimulation; and $F_{\text {pas }}$, the average force level before quick release without electrical stimulation.

The use of this normalization and correction scheme is based on previous work (van Mastrigt \& Tauecchio, 1982) and was justified by the results of variance analysis (see below). To support further the use of $F_{\text {rel }}$ (which implies a 'Maxwell' type configuration) as opposed to the use of $F_{\mathrm{d} l} / F_{\text {trig }}$ (which would follow from a 'Voigt' type configuration), and to provide a final test of independence of the conclusions drawn from this normalization, measurements were also processed using $F_{\mathrm{d}} / F_{\text {trig. All measured force }}$ curves were checked visually to ensure that the detected minima were not artefacts. Figure 2 shows an example of such a curve. The $F_{\text {rel }}$ values measured at one strip length (generally in two different contractions) were combined and fitted with the function:

$$
F_{\text {rel }}=A(\exp (b . d l)-1)+1-g \cdot d l
$$
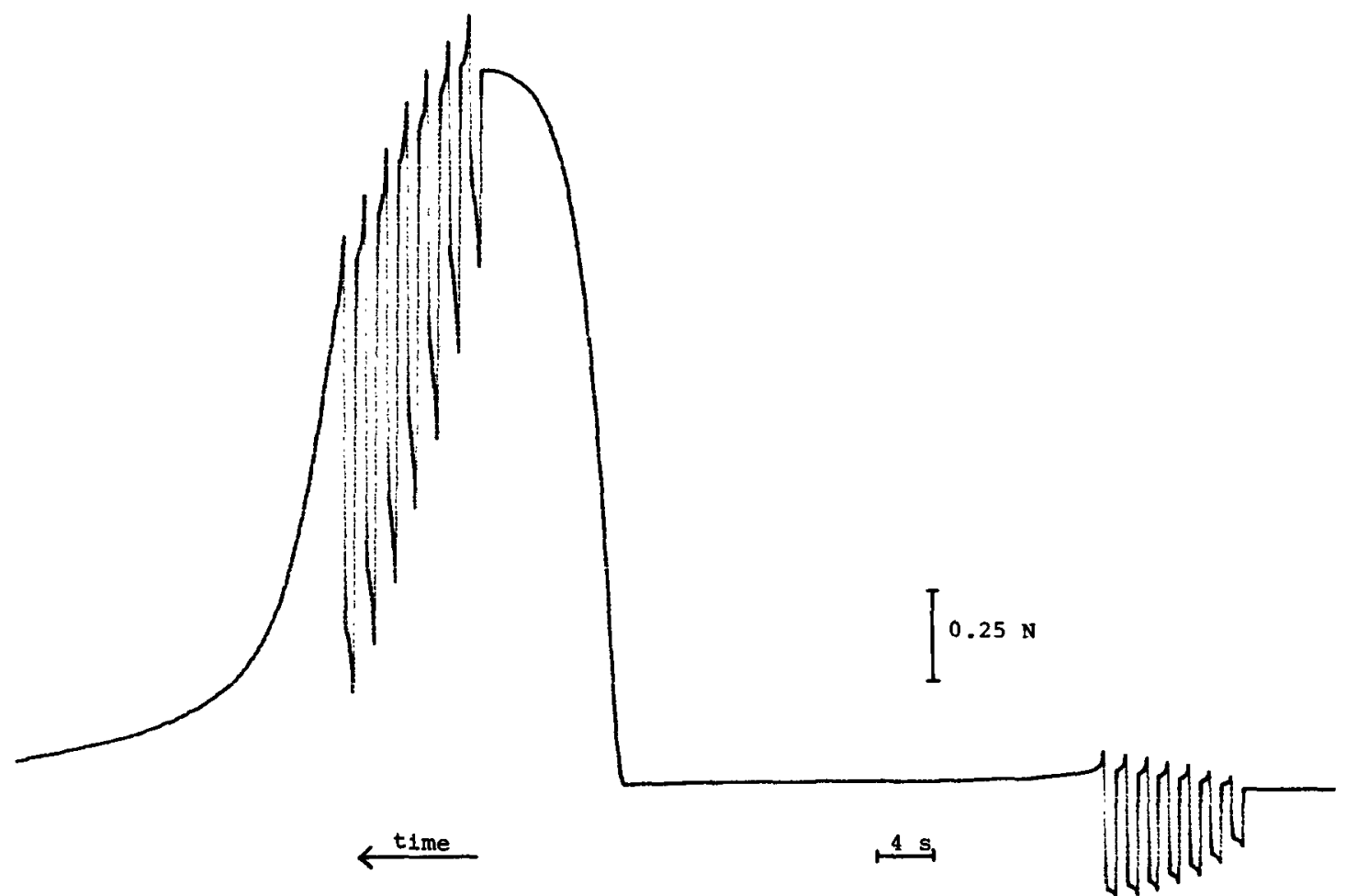

Fig. 1 Force as a function of time during one measurement with seven quick releases and resets applied, before and during contraction. 


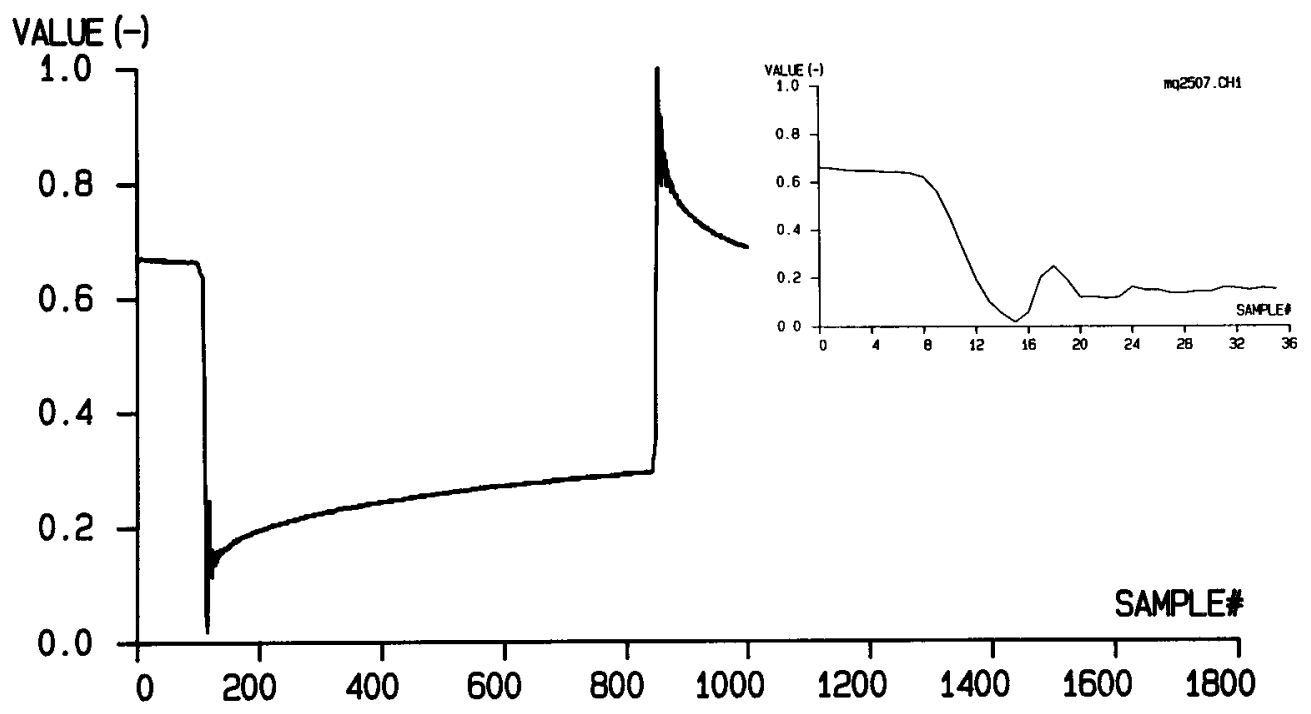

Fig. 2. Force as a function of time during quick release and reset. Inset shows $36 \mathrm{~ms}$ in greater detail.

where $d l$ is the amplitude of the quick release and $A, b$ and $g$ are parameters.

The function (2) was fitted using a stepwise approximation method (van Mastrigt, 1977). In the fitting procedure $A$ was limited to values smaller than or equal to 1 . Figure 3 shows an example of a fitted curve. The data were thus completely described in terms of the three parameters $A, b$ and $g$. As these parameters are difficult to interpret physiologically, the fitted functions were extrapolated to yield an estimate of the parameter $H$, the intercept of the function on the horizontal axis, which is the amplitude of the quick release necessary to reduce the active force to

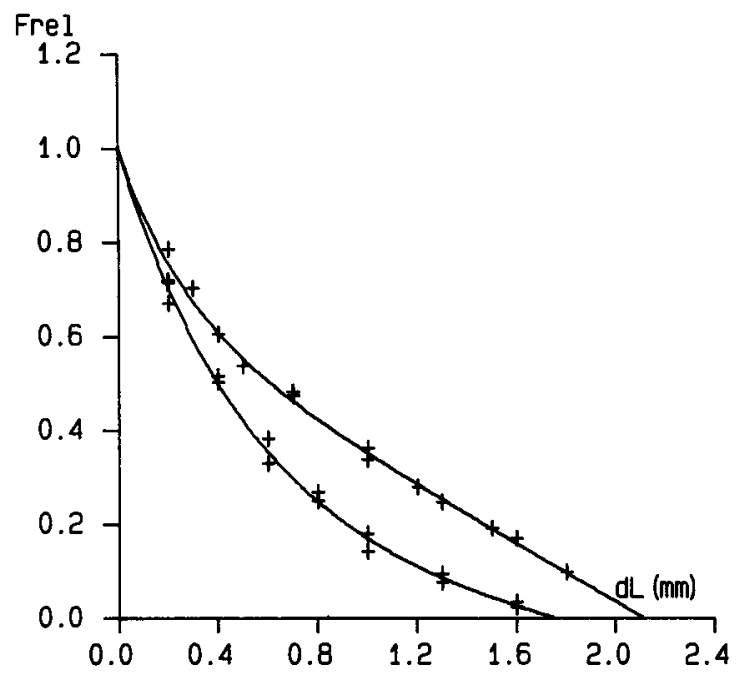

Fig. 3. The measured minimal active force during a quick release as a function of the quick release amplitude, and fitted with equation (2) (see text). Results with relative strip lengths 0.82 (lower curve) and 1.14 (upper curve) are shown. Both comprise data from two successive contractions. exactly zero (Huxley, 1957). Using this parameter, the curves measured at different stretched strip lengths were normalized, i.e. the quick release amplitudes were expressed as a percentage of $H$. Figure 4 shows on one graph all the normalized functions (2) measured on one strip at different stretched lengths. The varying curvatures of these functions were described by a second parameter:

$$
D_{\mathrm{b}}=0.7-F_{\text {rel }}(0.3 H)
$$

$D_{\mathrm{b}}$ represents the deviation of the fitted function from a straight line between the points $\left(F_{\text {rel }}=1.0, d l=0\right)$ and $\left(F_{\text {rel }}\right.$ $=0, d \mathrm{l}=1.0 \mathrm{H}$ ), measured at $d \mathrm{l}=0.3 \mathrm{H}$ (see Fig. 5). Thus if the fitted function were a straight line, $D_{\mathrm{b}}$ would be equal to 0 ; if the fitted function had the greatest possible curvature, $D_{\mathrm{b}}$ would be equal to 0.7 . The force curves measured during quick releases were thus characterized in terms of the physiological parameters $H$ and $D_{\mathrm{b}}$, instead of the

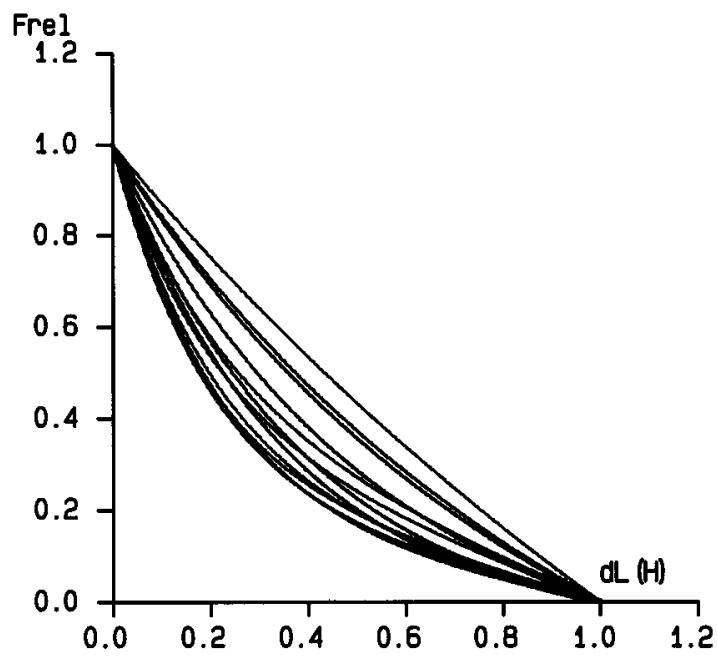

Fig. 4. All the fitted and normalized quick release functions from a single strip (at different stretched strip lengths). 


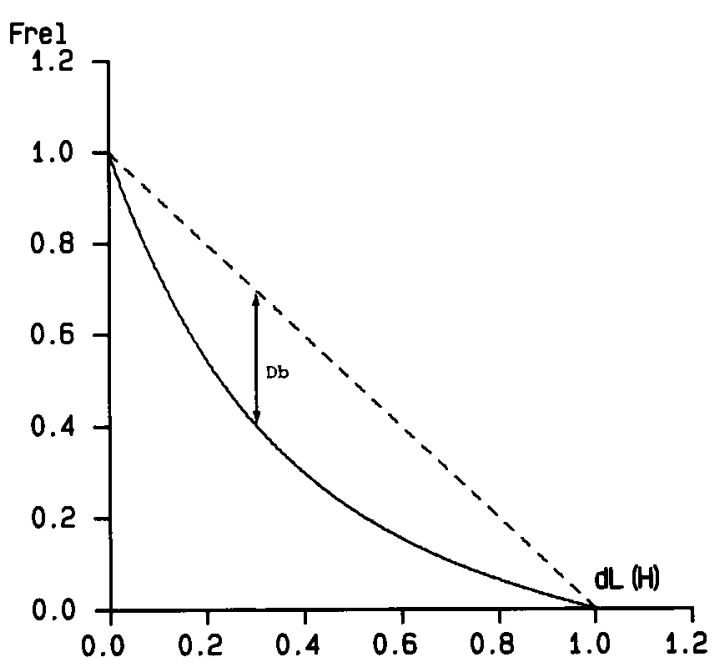

Fig. 5. Example of calculation of the parameter $D_{\mathrm{b}}$, which characterizes the degree of curvature of a fitted quick release function.

mathematical parameters $A, b$ and $g$. The strip lengths at which measurements were taken were normalized by dividing by the length at which the active force attained its maximum value. The resulting curves showing parameters $H$ and $D_{\mathrm{b}}$ (as well as the active force and the passive force) as functions of relative stretched length were interpolated to yield equidistant data points, which were averaged over all measured strips. The active force was also normalized at a given relative length by dividing it by the maximum active force attained in that strip.

\section{Results}

Measurements were performed on five strips. Figure 2 shows an example of the force measured during a quick release and reset. There is a short oscillatory phenomenon at the end of the release phase which is a mechanical artefact that was ignored in this study. Table 1 shows the results of variance analysis applied to $F_{\mathrm{dl}}$, the minimum force during the release (sample number 15 in the inset of Fig. 2), $F_{\mathrm{dl}} / F_{\text {trig }}$ and $F_{\text {rel }}$. In the latter two analyses variance is an order of magnitude smaller as compared to the analysis of $F_{\mathrm{dl}}$, indicating the significance of normalizing $F_{\mathrm{dl}}$ values with respect to $F_{\text {trig. There is no significant difference }}$ between the analyses of $F_{\mathrm{dl}} / F_{\text {trig }}$ and $F_{\text {rel }}$. In both cases almost all variance is explained by the factors $d l$ and strip length. $F_{\text {rel }}$ was used in all further calculations and analyses, except where explicitly indicated. Figure 3 shows this parameter as a function of $d l$, for all measurements made on one strip, at two strip lengths. Equation (2) was chosen to describe the curves on pragmatical grounds. The form of the curves (Fig. 3) suggests an exponential function, which can be understood theoretically (van Mastrigt \& Tauecchio, 1982), but a function with one
Table 1. Variance analyses of variables and combinations of variables used to describe the minimum force during quick release.

\begin{tabular}{|c|c|c|c|c|}
\hline Strip & Variable & $\begin{array}{l}\text { Mean squ } \\
d \mathrm{l}\end{array}$ & $\begin{array}{l}\text { are for source } \\
\text { length }\end{array}$ & $\begin{array}{l}\text { of variation: } \\
\text { residual }\end{array}$ \\
\hline 1 & $\begin{array}{l}F_{\mathrm{dl}} \\
F_{\mathrm{dd}} / F_{\text {trig }} \\
F_{\text {rel }}\end{array}$ & $\begin{array}{l}1.94 \\
0.58 \\
0.55(4)\end{array}$ & $\begin{array}{l}0.55 \\
0.023 \\
0.022(7)\end{array}$ & $\begin{array}{l}0.016 \\
0.00032 \\
0.00035(40)\end{array}$ \\
\hline 2 & $\begin{array}{l}F_{\mathrm{dl}} \\
F_{\mathrm{dl}} / F_{\text {trig }} \\
F_{\text {rel }}\end{array}$ & $\begin{array}{l}7.44 \\
0.74 \\
0.85(5)\end{array}$ & $\begin{array}{l}1.72 \\
0.018 \\
0.064(9)\end{array}$ & $\begin{array}{l}0.047 \\
0.00032 \\
0.00065(69)\end{array}$ \\
\hline 3 & $\begin{array}{l}F_{\mathrm{dl}} \\
F_{\mathrm{dd}} / F_{\text {trig }} \\
F_{\text {rel }}\end{array}$ & $\begin{array}{l}6.67 \\
0.80 \\
0.94(6)\end{array}$ & $\begin{array}{l}3.44 \\
0.065 \\
0.19 .(11)\end{array}$ & $\begin{array}{l}0.057 \\
0.00038 \\
0.0016 \quad(106)\end{array}$ \\
\hline 4 & $\begin{array}{l}F_{\mathrm{dl}} \\
F_{\mathrm{dl}} / F_{\text {trig }} \\
F_{\text {rel }}\end{array}$ & $\begin{array}{l}1.43 \\
0.45 \\
0.42(4)\end{array}$ & $\begin{array}{l}2.40 \\
0.095 \\
0.086(11)\end{array}$ & $\begin{array}{l}0.024 \\
0.00049 \\
0.0032 \quad(77)\end{array}$ \\
\hline 5 & $\begin{array}{l}F_{\mathrm{dl}} \\
F_{\mathrm{dl}} / F_{\text {trig }} \\
F_{\text {rel }}\end{array}$ & $\begin{array}{l}4.06 \\
0.76 \\
0.76(4)\end{array}$ & $\begin{array}{l}0.54 \\
0.016 \\
0.034(10)\end{array}$ & $\begin{array}{l}0.038 \\
0.00035 \\
0.00029(65)\end{array}$ \\
\hline
\end{tabular}

Values in parentheses are degrees of freedom.

exponential term, or one exponential term plus a constant, did not adequately fit the measured data. Furthermore, the extrapolation of such a function to yield the parameter $H$ can introduce large errors. Addition of a linearly decreasing term to the exponential term eliminates this problem, and was suggested by the almost linear tail of many measured curves especially at larger stretched strip lengths. By restricting the parameter $A$ to values smaller than or equal to 1, all measured curves could be satisfactorily fitted and reliably extrapolated. Figure 3 shows a typical example of the average goodness-of-fit of the curves. The sums of squared deviations for these examples were 0.0020 and 0.0040 respectively.

Figure 6 shows the average and the standard deviation (where available) of the normalized active force, passive force and total force as a function of the relative length. Average unnormalized active force at a relative length of 1.0 was approximately $2.5 \mathrm{~N}$, implying an active stress in the order of $40 \mathrm{mN} \mathrm{mm}^{-2}$. At relative lengths greater than 1.0 the passive force becomes very large in these preparations, so that permanent deformation of strips cannot be excluded (Griffiths et al., 1979; van Mastrigt \& Glerum, 1985). Figure 7 shows that $H$ divided by the stretched strip length is approximately constant, so that $H$ is roughly proportional to the relative length.

The curves showing the minimum active force during a quick release (Fig. 3) change significantly in shape when the strips are stretched: at greater stretched lengths the curve tends to approach a 


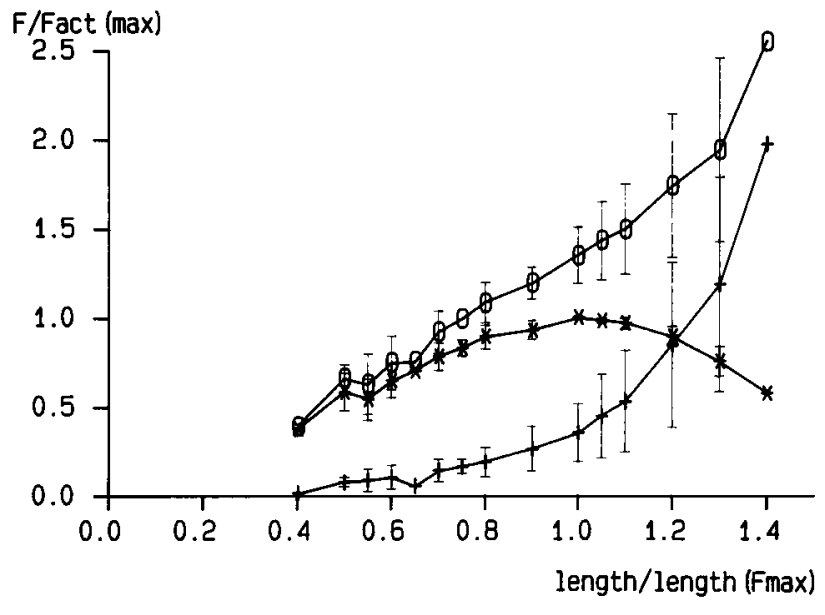

Fig. 6. Average plus and minus standard deviation of normalized active, passive and total force as a function of relative stretched strip length.

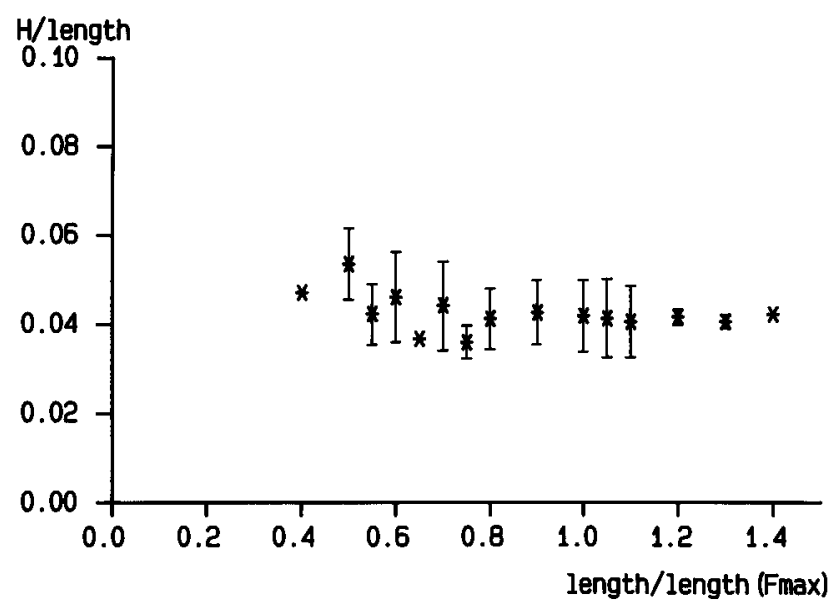

Fig. 7. The parameter $H$ divided by stretched strip length as a function of relative stretched strip length. Average plus and minus standard deviation.

straight line (see also Fig. 4). This behaviour is quantified by the $D_{\mathrm{b}}$ parameter in Fig. $8\left(D_{\mathrm{b}}=0\right.$ signifies a straight line). It can be seen that, especially at relative lengths greater than $1.0, D_{\mathrm{b}}$ decreases. The $D_{\mathrm{b}}$ values at length/length $\left(F_{\max }\right)=1.3$ and 1.2 differ significantly from the value at 0.5 (Students $t$-test, $P<$ 0.05).

\section{Discussion}

Although the structure of smooth muscle is not as well understood as that of striated muscle, smooth muscle contraction certainly involves a sliding of actin and myosin filaments past each other accompanied by the successive formation, (apparent) shortening and release of cross-bridges between the filaments (Gabella, 1981; Bagby \& Corey-Kreylin,

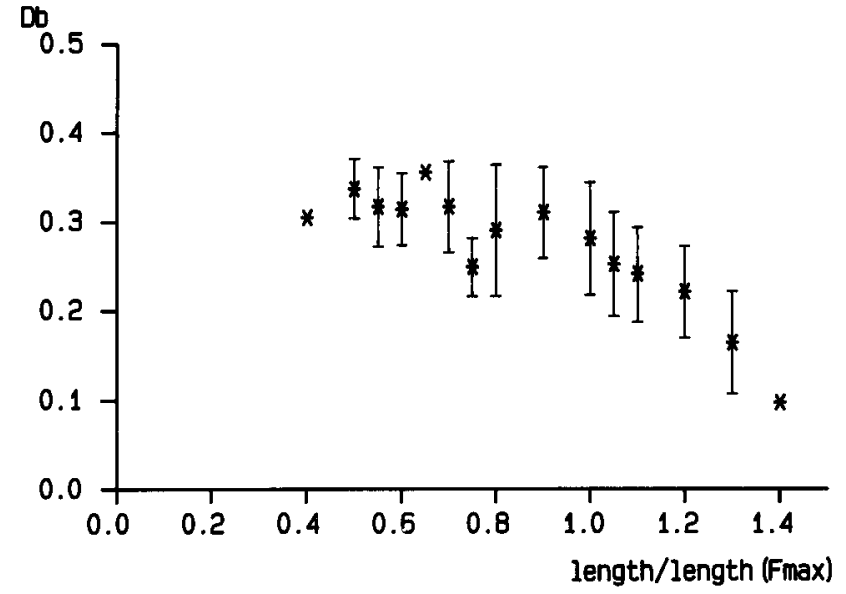

Fig. 8. The parameter $D_{\mathrm{b}}$ as a function of stretched strip length. $D_{\mathrm{b}}$ signifies the shape of the curves of minimal active force during quick release. $D_{\mathrm{b}}=0$ signifies a straight line. Average plus and minus standard deviation of $D_{\mathrm{b}}$ is displayed.

1984). A macroscopic piece of smooth muscle in this view consists of a network of cells, each containing a network of filaments. As a result, the macroscopically measured force is the sum of the forces generated by many cross-bridges in parallel and the macroscopic shortening is the sum of the shortening of many cross-bridges in series. Traditionally, and for striated muscle, the dependence of active force on stretched strip length has been ascribed to a change in the degree in overlap of filaments (Huxley, 1974). For smooth muscle too it has been postulated that changes in length primarily change the overlap of filaments, thus influencing the number of crossbridges that can be formed (Bagby \& Corey/Kreyling, 1984). According to this concept, the force generated at a given muscle length is directly proportional to the number of active cross-bridges in parallel between filaments. The parameter $H$ studied in our measurements represents the shortening necessary to reduce the active force instantaneously to exactly zero. This parameter thus describes the working range of the muscle and would, in the most simple model, represent the product of the average working range of a cross-bridge with the effective number of crossbridges in series, which is equal to the number of filaments connected in series. Taking this view, the addition of more cross-bridges in parallel between an invariable number of filaments should not influence this working range of the muscle. The normalization applied in equation (1), which allows quick releases measured at different active force levels during one, or even several, contraction(s) to be plotted on a single curve (Fig. 3), depends on this assumption which is based on earlier work (van Mastrigt \& Tauecchio, 1982). The same equation also implies the additivity of passive and active force. Both assump- 
tions basically describe a Maxwell configuration of elasticity: series elasticity in series with a contractile element, and a passive elasticity in parallel to both. As an alternative a series elasticity can be thought of which is in series with both the contractile element and the parallel passive elasticity: a Voigt configuration. Both configurations have been proposed for smooth muscle (Ford et al., 1977; Bressler \& Clinch, 1974). The additivity of active and passive force has been demonstrated for this type of tissue using a different type of measurement (Griffiths et al., 1979). In the present data there is no significant difference in explained variance versus residual variance between analyses according to the Voigt model, where normalization should exclude passive force as in the variable $F_{\mathrm{d} /} / F_{\text {trig }}$ (Table 1) and the Maxwell model where it should be included as in $F_{\text {rel }}$. Nor did a significant difference exist between the forms of the quick release curves for both parameters, except that analysis on the basis of the variable $F_{\mathrm{d} 1} / F_{\text {trig }}$ yielded a lower $H$ value as compared to analysis based on $F_{\text {rel }}$. Figure 9 shows a replot of part of the data of Fig. 3 for the former analysis method. It is concluded that neither of the models is strictly correct.

For compatibility with earlier work (van Mastrigt \& Tauecchio, 1982) the Maxwell type of analysis using equation 1 was maintained. Assuming that increasing stretched strip length cannot increase the number of cross-bridges in series, normalized quick release results (and thus the resulting parameter $H$ ) should not be affected by changes in active force caused by changes in stretched strip lengths. In our data, the parameter $H$ is shown to be not only dependent on, but even proportional to, stretched strip length (Fig. 7) even though the active force is not (Fig. 6). If we

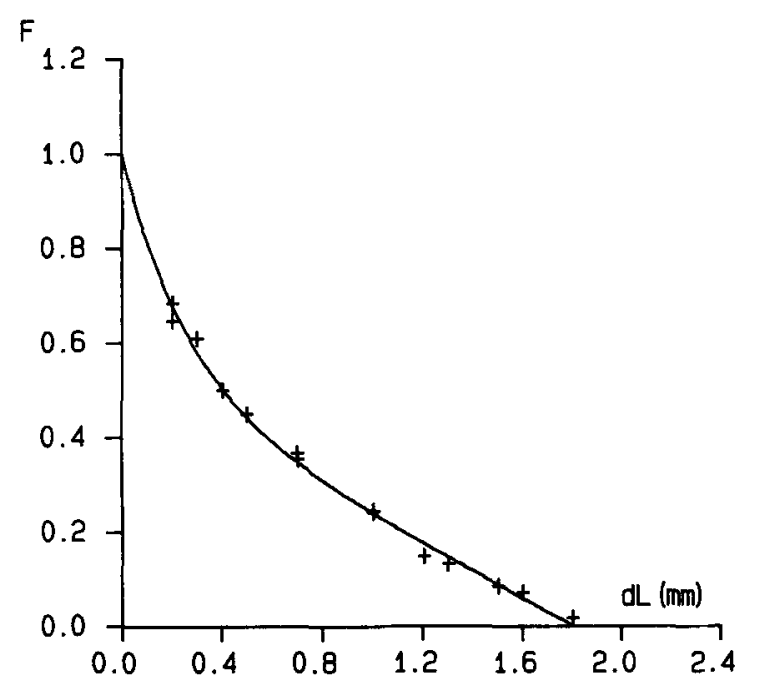

Fig. 9. Replot of one curve from Fig. 3, for the parameter $F_{\mathrm{d} 1} / F_{\text {trig }}$ as opposed to $F_{\text {rel }}$. continue to make the assumption that the working range of the muscle results from the summation of cross-bridges in series, the observed dependence can only be understood if the filaments are not oriented longitudinally. Stretching the strip then causes filaments to take a more longitudinal orientation, so changing the macroscopically measured parameter $H$. Indeed, filaments within smooth muscle cells do not run longitudinally, so that a reorientation upon stretching or shortening is to be expected (Fay et al., 1984). It is shown in the appendix that the change in $D_{\mathrm{b}}$ which can be expected from a reorientation of filaments from randomly orientated to fully longitudinally orientated is of the order of 0.13 , on the assumption that the quick release curve for each filament (the microscopic equivalent of Fig. 3) does not greatly deviate from a straight line. As the minimum $D_{\mathrm{b}}$ value which was measured at the largest relative length $\left(D_{\mathrm{b}}=0.05\right.$, strip 4$)$ does not differ greatly from zero, this assumption appears to be valid. Figure 8 shows that the value of $D_{\mathrm{b}}$ averaged over the five measured strips changes significantly more than 0.13. In two individual strips the change in $D_{\mathrm{b}}$ on stretching was more than twice this value. It can therefore be concluded that reorientation of filaments is not the primary cause of the changes observed on stretching.

An alternative explanation for the dependence of shortening behaviour on stretched length is that a significant part of the measured series elasticity is due to an extra external passive elasticity in series with the cross-bridges. Although this idea has been suggested previously (Mulvany, 1984; Hellstrand \& Johansson, 1979; Hellstrand, 1979; Meiss, 1978) accurate determinations of the relative size of the contribution of passive series elasticity to the total series elasticity has been lacking. Figure 10 shows a direct plot of the parameter $H$ against the total force (active + passive) for all strips, which indicates clearly that a unique relation exists between these two variables. This relation can be thought of as the sum of two characteristics. A constant representing the series elastic characteristic of the cross-bridges: whatever the force, a constant shortening $H$ must be applied to exactly zero it, and a characteristic for the passive series elasticity which must be linear, i.e. the higher the total force, the more shortening must be applied to zero it. The extrapolation of the data in Fig. 10 yields a vertical intercept of $H=0.99 \mathrm{~mm}$ which therefore represents the characteristic of the crossbridges. Since the average initial length of the five strips was $26 \mathrm{~mm}, H /$ initial length for the cross-bridge elasticity is $3.8 \%$. For other types of smooth muscle, values of $H /$ initial length of the same order or less have been reported: $4-5 \%$ for rabbit mesotubarium (Meiss, 1978); 1-2\% for rabbit urinary bladder (Hellstrand \& Johansson, 1979; Hellstrand, 1979); $1.5 \%$ for 


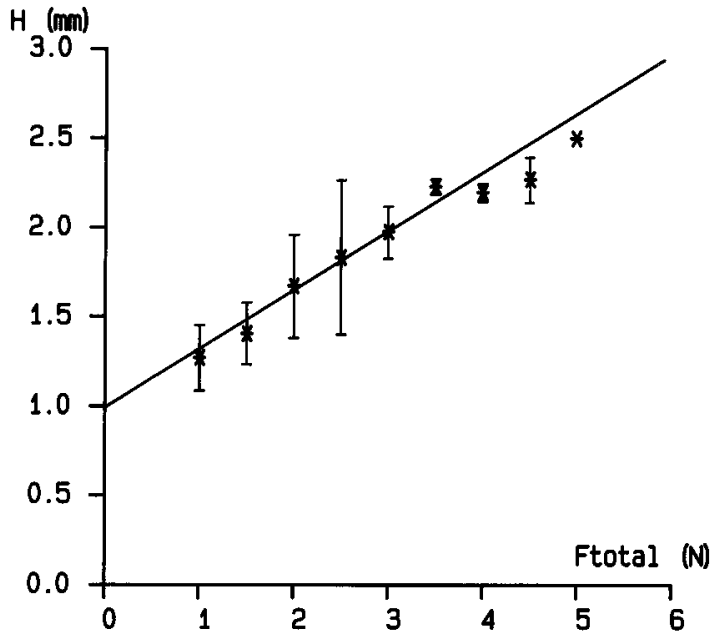

Fig. 10. Average plus and minus one standard deviation of the parameter $H$ (the amplitude of quick release necessary to reduce active force to exactly zero) as a function of total force (active force + passive force) for five strips, and fitted straight line.

single cell measurements on Bufo marinus stomach (Warshaw \& Fay, 1984).

In Warshaw and Fay's work (1984), quick release curves were linearly extrapolated, which probably yields an underestimate of the value of $H /$ initial length. These values of $H /$ length are about 10 times larger than the estimates of cross-bridge elastic extension for striated muscle (Ford et al., 1977) which points to a drastic difference in structure (shorter filaments and/or more compliant cross-bridges). Figure 10 also shows that the passive series elasticity is almost linear. Fitting a straight line yields an elastic coefficient of $3.1 \mathrm{~N} \mathrm{~mm}^{-1}$. The form of this curve does not depend on the usage of the parameter $F_{\text {rel }}$ as opposed to using $F_{\mathrm{d} l} / F_{\text {trig. }}$. Recalculating Fig. 10 using the latter parameter yields a similar form, a correlation coefficient of 0.80 (whereas Fig. 10 yields 0.79 ) an intercept of $0.78 \mathrm{~mm}$ as opposed to $0.99 \mathrm{~mm}$ and a slope of $3.8 \mathrm{~N} \mathrm{~mm}^{-1}$. The reduction in the curvature of the measured quick release curves on stretching (see Figs 5 and 8) can thus be accounted for by the increasing relative importance of the linear passive series elasticity and the descreasing relative importance of the non-linear cross-bridge series elasticity as the muscle strip is stretched. The form of the cross-bridge elasticity can be estimated from Fig. 9 by extrapolating the parameter $D_{\mathrm{b}}$ back to zero extension. This yields a value for $D_{\mathrm{b}}$ of the order of 0.3 .

\section{Appendix}

Suppose filaments are randomly orientated in the preparation, i.e. the angle $a$ between filaments and a plane perpendicular to the direction in which force is measured is distributed uniformly in the range 0 $\pi / 2$. The macroscopically measured force $F$ can then be calculated from the forces $f$ in each filament as:

$$
F=\int_{0}^{\pi / 2} f \cdot \sin (a) \mathrm{d} a
$$

The force in a filament $f$ depends on the amplitude of a quick shortening in the filament $d \mathrm{l}$. Suppose this dependence (the microscopic equivalent of the relation depicted in Fig. 3) takes the form of a straight line between $f=1, d \mathrm{l}=0$ and $f=0, d \mathrm{l}=h$ ( $h$ is the per filament equivalent of the macroscopic parameter $H$ ), and zero for $d \mathrm{l} / h$. $f$ can then be written as:

$$
f=(1-d \mathrm{l} / h) \cdot(1-u(d \mathrm{l}-h))
$$

where $u()$ represents the unit function. Furthermore, the relation between a macroscopically applied shortening $d \mathrm{~L}$ and the resulting shortening of the filaments $d \mathrm{l}$ can be described as:

$$
d \mathrm{~L}=d l \cdot \sin (a)
$$

Combining 4,5 and 6 yields $F(d \mathrm{~L})$, the macroscopically measurable quick release function. This function is not a straight line, and its curvature can be calculated by normalizing it (dividing by $F(0)$ ), inserting it into equation 3 and integrating. This yields $D_{\mathrm{b}}=0.13$. Now, if by extreme stretching the orientation of filaments changes such that all filaments lie in the direction in which force is measured, the macroscopic function $F(d \mathrm{~L})$ would equal the microscopic $f(d l)$ apart from a scale factor. As $f(d l)$ is a straight line the curvature of $F(d \mathrm{~L})$ would yield $D_{\mathrm{b}}=0$. The change in curvature of $F(d \mathrm{~L})$ by an extreme reorientation of filaments, from randomly orientated to exclusively longitudinally orientated thus leads to a change in $D_{\mathrm{b}}$ from 0.13 to 0 , on the assumption that the per filament $f(d l)$ function is a straight line. For other $f(d l)$ functions, not deviating too much from a straight line, the maximal change in $D_{\mathrm{b}}$ due to fibre reorientation will be of the same order of magnitude.

\section{References}

BAGBY, R. M. \& COREY-KREYLING, M. D. (1984) Structural aspects of the contractile machinery of smooth muscle: 'Is the organization of contractile elements compatible with a sliding filament mechanism?' In Smooth Muscle Contraction. (Edited by STEPHENS, N. L.) pp. 47-74. New York: Marcel Dekker.
BRESSLER, B. H. \& CLINCH, N. F. (1974) The compliance of contracting skeletal muscle. J. Physiol (Lond.) 237, 477-93.

FAY, F. S., FOGARTY, K. \& FUJIWARA, K. (1984) The organization of the contractile apparatus in single isolated smooth muscle cells. In Smooth Muscle Contrac- 
tion. (edited by STEPHENS, N. L.) pp. 75-90. New York: Marcel Dekker.

FORD, L. E., HUXLEY, A. F. \& SIMMONS, R. M. (1977) Tension responses to sudden length change in stimulated frog muscle fibres near slack length. J. Physiol. (Lond). 269, 441-515.

GABELLA. G (1981) Structure of smooth muscles. In Smooth Muscle: An Assessment of Current Knowledge (edited by BULBRING, E. et al.) pp. 1-46. London: Edward Arnold.

GRIFFITHS, D. J., VAN MASTRIGT, R., VAN DUYL, W. A. \& COOLSAET, B. L. R. A (1979) Active mechanical properties of the smooth muscle of the urinary bladder. Med. Biol. Eng. Comp. 17, 281-90.

HELLSTRAND, P. \& JOHANSSON, B. (1979) Analysis of the length response to a force step in smooth muscle from rabbit urinary bladder. Acta Physiol. Scand. 106, 221-38.

HELLSTRAND, P. (1979) Mechanical and metabolic properties related to contraction in smooth muscle. Acta Physiol. Scand Suppl. 464.

HUXLEY, A. F. (1957) Muscle structure and theories of contraction. Progr. Biophys.Chem. 7, 255-318.

HUXLEY, A. F. (1974) Review Lecture: Muscular Contraction. J. Physiol (Lond). 243-1, 1-43.

KREBS, H. A. (1932) Untersuchungen uber die harnstoffbildung in Tierkorper. Hoppe Seyler's Zeit. Physiol. Chemie 210, 33-67.

MEISS, R. A. (1978) Dynamic stiffness of rabbit mesotubar- ium smooth muscle: Effect of isometric length. Am. J. Physiol 234-1, C14-C26.

MULVANY, M. J. (1984) Cross-bridges in smooth muscle. In Smooth Muscle Contraction, (edited by STEPHENS, N. L.) pp. 145-50. New York: Marcel Dekker.

MURPHY, R. A. (1976) Contractile system function in mammalian smooth muscle. Blood Vessels 13, 1-23.

VAN MASTRIGT, R. (1977) Constant-step approximation of multi-exponential signals using a least squares criterion. Comp. Biol. Med. 7, 231-47.

VAN MASTRIGT, R. \& TAUECCHIO, E. A. (1982) Serieselastic properties of strips of smooth muscle from pig urinary bladder. Med. Biol. Eng. Comp. 20, 585-94.

VAN MASTRIGT, R. \& GLERUM, J. J. (1985) Electrical stimulation of smooth muscle strips from the urinary bladder of the pig. J. Biomed. Eng. 7, 2-8.

VAN MASTRIGT, R., KOOPAL, J. W. B., HAK, J. \& VAN DE WETERING, J. (1986) Modeling the contractility of urinary smooth muscle using isometric contractions. Am. J. Physiol. 251, R978-R983.

MEISS, R. A. (1978) Dynamic stiffness of rabbit mesotubarium smooth muscle: effect of isometric length. Am.J. Physiol. 234-1, C14-C26.

WARSHAW, D. M. \& FAY, F. (1984) Tension transients in single isolate smooth muscle cells: insight into the cross-bridge mechanism. In Smooth Muscle Contractions (edited by STEPHENS, N. L.) pp. 131-44. New York: Marcel Dekker. 